\title{
Levy Walk Based Multi-Hop Data Forwarding Protocol for Opportunistic Mobile Phone Sensor Networks
}

\author{
Thejaswini $\mathrm{M}^{\dagger}$, P. Rajalakshmi ${ }^{\dagger \dagger}$, U. B. Desai ${ }^{\dagger \dagger}$ \\ ${ }^{\dagger}$ Department of Computer Science and Engineering \\ ${ }^{\dagger}$ Department of Electrical Engineering \\ Indian Institute of Technology Hyderabad, India \\ Email: (cs10p003, raji, ubdesai)@iith.ac.in
}

\begin{abstract}
Unstable link connectivity due to dynamic mobility nature of mobile phone users and error prone wireless link quality increases end-to-end delay for mobile phone based opportunistic network applications. This problem becomes more worse in the presence of large amount of data transmission, like multimedia data. This paper refers to Levy walk based multihop data forwarding protocol called Data Transmission Time and Human Walk Velocity (DTT-HWV) for Opportunistic Mobile Phone Sensor Networks (OMPSN). This paper, in particular evaluates the performance of proposed protocol in terms of endto-end waiting time to receive data, which is an important QoS requirement for data transmission in opportunistic networks. The proposed protocol DTT-HWV reduces end-to-end waiting time to receive data compared to Random Progress (RP) data forwarding method in presence of low battery power and high path loss. Obtained results are helpful in designing and building of large scale data retrieval services for opportunistic networks involving humans in the communication network loop.
\end{abstract}

\section{INTRODUCTION}

Smart phones embedded with rich media sensors (camera and microphones) are initiating development of ubiquitous mobile sensing applications [1]. As smart phones are attached to human users, their unpredictable mobility movements create an opportunistic network [2] scenario which usually lacks presence of end-to-end path for routing data between source and destination mobile phone devices. Transmission of large scale multimedia sensor data (audio, video) is usually affected by unstable routes caused by topological dynamic mobile networks. This paper discusses multi-hop data forwarding protocol for Opportunistic Mobile Phone Sensor Networks (OMPSN) based on Levy walk (LW) [3], [4], [5] mobility model. We assume mobile phones communicate with each other using IEEE 802.11 ad-hoc mode [6]. Proposed data forwarding protocol is applicable to scenarios like disaster relief and military activity fields, where infrastructure or centralized data communications are not available to use.

Random waypoint (RWP) [7], [8] is the standard mobility model used in most of the research work for evaluating the performance of routing protocols in mobile networks. Unlike LW, RWP mobility model does not depict the statistical similarities with human mobility patterns, so LW has been a choice for realistic mobile networks simulation [3], [4], [5],
[9], [10]. The analytical description of LW mobility model is given in section $\mathrm{II}(\mathrm{C})$. We use LW mobility model given in [9] for the simulation of proposed work. Human intercontact times (ICT) follows power-law distributions [3], [4], [5]. Under Truncated Levy Walk (TLW), which is a simple LW mobility model, the impact of heavy-tail flight distribution on routing protocols for mobile networks is discussed in paper [3] and [4]. Heavy-tail flight distribution of LW results in heavytail delays for mobile routing protocols [3]. As LW mobility parameter $\alpha$ value decreases (section II(C), $0<\alpha<2$ ), mobile networks becomes more dynamic and vice-versa [4].

In this paper we evaluate the contact opportunities in terms of expected waiting time to get a neighbor mobile node (or frequency of contacting a neighbor mobile node). In particular we analyse the expected end-to-end waiting time to receive data in OMPSN, constrained over application, network and LW mobility model parameters. Geographic or location aware data forwarding [11], [12] techniques is used when the network topology is dynamically changing. We propose a simple data forwarding protocol based on data transmission time and human walk velocity called DTT-HWV. We compare proposed data forwarding protocol with random progress (RP) [12], [13] data forwarding method, where data sending node selects randomly one of its neighbor node which is having forward progress and overcomes the trade off between progress and transmission success. The simulation results shows that DTTHWV performs better than RP data forwarding method in terms of end-to-end waiting time to receive data in OMPSN.

The remainder of this paper is organized as follows. Section II describes system model for the proposed work. Section III gives description of proposed work. Section IV discusses evaluation of simulation results and Section $\mathrm{V}$ concludes the paper.

\section{SySTEM MODEL}

This section provides the description of models used for the development of proposed work. 


\section{A. Radio Propagation Model}

Friis free space equation [14] is given in equation (1), where $P_{r}(d)$ is received power at distance $d$ from the transmitting source node. $P_{t}, G_{t}$ and $G_{r}$ are transmitted power, transmitter gain and receiver gain respectively. $\lambda$ is wavelength of transmitted wave.

$$
P_{r}(d)=P_{t} G_{t} G_{r} \frac{\lambda^{2}}{(4 \pi d)^{2}}
$$

\section{B. Received Signal Strength Model}

The equation to obtain received signal strength $(R S S)$ in $\mathrm{dB}$ [14] is given in equation (2), where $P_{r}\left(d_{0}\right)$ is received power at reference distance $d_{0}$ in $\mathrm{dB}$ and $\eta$ is path loss exponent parameter.

$$
R S S=P_{r}\left(d_{0}\right)+10 \eta \log \frac{d_{0}}{d}
$$

\section{Levy-Walk Mobility Model}

Human walk patterns closely follow LW patterns [3]. LW are optimal way to search targets which is similar to animal foraging patterns [15]. A step in LW mobility model is represented by four variables $\left(l, \theta, \Delta t_{f}, \Delta t_{p}\right)$ [3], [4]. Flight is defined as straight line trip without direction change or pause. $l$ is length of flight, drawn from power law distribution with parameter $\alpha$ and its probability density function [3] is given in equation (3). $l_{\min }$ and $l_{\max }$ are minimum and maximum flight length. At the beginning of each flight, LW node chooses a direction with angle $\theta$ randomly from a uniform distribution within range $[0,2 \pi] . \Delta t_{f}[3]$ is flight time, the time taken by LW nodes to complete the flight length and is given in equation (4). $\kappa$ and $\varrho$ are constants. $\Delta t_{p}$ is pause time, drawn from power law distributions with parameter $\beta$ and its probability density function [3] is given in equation (5). $t_{p \min }$ and $t_{p \max }$ are minimum and maximum pause time. The levy distribution [3] is given in equation (6), where $s$ and $a$ are scale factor and exponent parameter respectively.

$$
\begin{gathered}
p(l) \propto \frac{1}{|l|^{(1+\alpha)}}, l_{\min }<l<l_{\max }, 0<\alpha<2 \\
\Delta t_{f}=\kappa l^{(1-\varrho)}, 0 \leq \varrho \leq 1 \\
p\left(\Delta t_{p}\right) \propto \frac{1}{\left|\Delta t_{p}\right|^{(1+\beta)}}, t_{\text {min }}<\Delta t_{p}<t_{\text {max }}, 0<\beta<2 \\
f_{X}(x)=\frac{1}{2 \pi} \int_{-\infty}^{+\infty} e^{-i t x-|s t|^{a}} d t
\end{gathered}
$$

\section{Battery Power Drain model}

Battery power drain of mobile phones depends on individual user usage patterns. We considered typical mobile phone applications like talking, audio playback, video playback, web browsing, idle time and their power usage rating [6], [16] for obtaining minimum and maximum battery power $\left(\gamma_{\min }\right.$ and $\gamma_{\max }$ ) drain range, at regular intervals of time $b t . \gamma$ is chosen randomly within the range $\left[\gamma_{\min }, \gamma_{\max }\right]$. We propose a battery power drain rate model, described in equation (7) and (8). $B_{b t_{2}}$ is battery power at time $b t_{2}$ and $B_{b t_{1}}$ is residual battery power at time $b t_{1}\left(b t_{2}>b t_{1}\right)$. Equation (7) gives battery power consumed for transmitting or receiving of data $\left(B_{t x \_r x}\right)$ and battery power consumed for GPS activity $\left(B_{G P S}\right)$.

$$
\begin{array}{r}
B_{F \text { data }}=B_{t x \_r x}+B_{G P S} \\
B_{b t_{2}}=B_{b t_{1}}-\left(\gamma+B_{F \text { data }}\right)
\end{array}
$$

To evaluate the proposed work the following assumptions are made. OMPSN consists of an IEEE 802.11 single channel ad-hoc network of mobile phones [14], [17] (mobile phones also called as mobile nodes). Let $N$ be the number of mobile nodes in a communication network area $A$. Communication range of mobile nodes is given by $r$. Simulation duration is represented by $d_{t}$. We consider two regions inside area $A$ called as Target $_{\text {region }}$ and Data $a_{\text {region }}$, given by their center location points and range. Our objective is to analyse end-to-end waiting time, which is the time taken to send

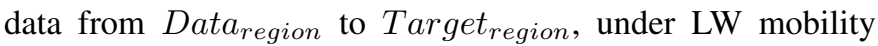
model and is represented by term TRRT (Target region $_{\text {Reach }}$ Time). For simplifying the performance evaluation of proposed work, we consider only single source data transmission event. We use parameters $\Delta, L Q_{\text {threshold }}$ and $B P_{\text {threshold }}$, where $r-\Delta$ distance is used to find mobile nodes which have the possibility of moving out of communication range during data transmission, $L Q_{\text {threshold }}$ is the threshold duration to sustain bad link quality and $B P_{\text {threshold }}$ is the required threshold battery power for a mobile node. We assume relay node will not forward data to its neighbor node (next relay node) until it receives complete data successfully and data transmission time which is in order of several seconds. We assume that mobile phones are embedded with GPS for location information and media sensors.

\section{Proposed Data Forwarding Protocol DESCRIPTION}

This section describes expected time to get a neighbor and proposed data forwarding protocol under LW mobility model.

\section{A. Expected Waiting Time to Get a Neighbor Node Under LW Mobility Model}

Low node density and low communication range both parameters have a negative impact on end-to-end delay in opportunistic mobile networks. For OMPSN, it is very difficult to provide appropriate end-to-end waiting time to receive data, as mobile phone users mobility is unpredictable. To analyse TRRT, we require expected time to get a new neighbor $\left(E_{N}\right)$ node to forward data. $E_{N}$ is calculated for given $N, A$, $d_{t}$, and $r$. Location trajectory of node $i(i=1,2, . ., N)$ is recorded for time $t_{i}$ at regular interval and corresponding counter variable $n$ value is incremented whenever it meets a new node (node which is not visited perviously) within its range. $E_{N}$ is calculated using equations (9), (10) and (11). $t_{i}(n)$ is the time when $i^{t h}$ node meets $n^{t h}$ new node. $E_{i}$ is the $i^{t h}$ node expected waiting time to get its neighbor node.

$$
W T_{i}= \begin{cases}t_{i}(n)-t_{i}(n-1), & \text { if } n>1 ; \\ t_{i}(1), & \text { if } n=1 ;\end{cases}
$$




$$
\begin{gathered}
E_{i}=\mathbb{E}\left(W T_{i}\right)=\sum_{n=1}^{N-1} \frac{W T_{i}}{n} \\
E_{N}=\mathbb{E}\left(E_{i}\right)=\sum_{i=1}^{N} \frac{E_{i}}{N}
\end{gathered}
$$

\section{B. One-Hop Waiting Time $\left(W_{t}\right)$}

We assume fixed packet size. The time required for a data packet to reach the one-hop neighbor node [17] is given by $T_{p_{d}}$ (packet delivery time). Overall packet delivery time between pair of mobile nodes is given in equation (12) which gives required minimum inter-contact time $\left(I C T_{\min } t x\right)$. We ignore RTS/CTS and small control packets (to exchange location, battery power information) transmission time as we consider data transmission for several seconds between pair of mobile nodes. $W_{t}$ is one-hop waiting time to receive overall packets, $T_{t_{x}}$ is transmission time, $T_{p_{p}}$ is propagation time and $O_{p}$ is total number of packets.

$$
\begin{aligned}
T_{p_{d}} & =T_{t_{x}}+T_{p_{p}} \\
W_{t} & =O_{p} * T_{p_{d}} \\
& =I C T_{\text {min_tx }}{ }
\end{aligned}
$$

\section{Expected Total Waiting Time $\left(E T W_{t}\right)$}

The expected total waiting time $\left(E T W_{t}\right)$ required for all the data packets to reach the Target region $_{\text {[17] is given in }}$ equation (13). $h$ is minimum number of hops, $T_{p_{s}}$ is processing time and $E_{N}$ is time spent by nodes after receiving overall data packets, and the time within which LW nodes are expected to get a new neighbor (equation (11)) for given $N, A, d_{t}$ and $r$. $h=\frac{D}{r}$, where $D$ is distance between center of Target region and center of Data $a_{\text {region }}$ and $r$ is range of mobile node. From equation (13), when $E_{N}$ is minimum say $E_{N} \approx 0$, minimum end-to-end waiting time $\left(T W_{t_{-} \text {min }}\right)$ bound for users to receive data is obtained from equation (14).

$$
\begin{array}{r}
E T W_{t}=h *\left(I C T_{\text {min_tx }}+T_{p_{s}}+E_{N}\right) \\
T W_{t \_m i n} \approx h *\left(I C T_{\text {min_t }}+T_{p_{s}}\right)
\end{array}
$$

\section{Proposed Data Forwarding Protocol DTT-HWV for OMPSN}

The communication range $r$ of mobile node is divided into several regions (Figure 1) based on data transmission time and velocity of human walk (DTT-HWV). We assume maximum human walk velocity is one meter/second [18] and data transmission time (in order of several seconds) is equal to $I C T_{\text {min_tx }}$ (equation (12)). For example, if $I C T_{m i n \_t x} \approx 19$ seconds, then radius of a region is equal to 19 meters. To simplify the algorithm, $I C T_{\text {min_tx }}$ should be less than $\frac{r}{2}$. The range of Region 1 and Region 3 are equal to $I C T_{\min \_t x}$. The three regions area and their range are given in equation (15), (16) and (17).

$$
\begin{aligned}
& \text { Region } 1=\pi * I C T_{\text {min_tx }}^{2}, \\
& 0<R e g i o n 1 \leq I C T_{\text {min_tx }}
\end{aligned}
$$

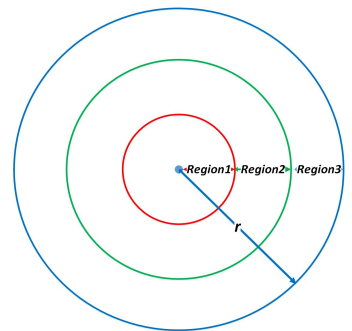

Fig. 1. Division of radio range of a node into three regions

\begin{tabular}{|c|c|}
\hline Simulation area $A$ & $1020 \mathrm{~m} * 1020 \mathrm{~m}$ \\
Simulation duration $d_{t}$ & 1020 seconds \\
Flight parameter $\alpha$ & 1.0 \\
Pause parameter $\beta$ & 1 \\
Average flight length & $5-1020$ meters \\
Pause time & $3-6$ seconds \\
Mobile node density $N$ & $100-700$ \\
Location sample interval & $1 \mathrm{~second}$ \\
Frequency (IEEE 802.11 ) & $2442 \mathrm{MHz}$ \\
Transmission power (IEEE 802.11 ) & $15 \mathrm{dBm}$ \\
Receiver sensitivity & $-68 \mathrm{dBm}$ \\
Path loss exponent value $\eta$ & 2 \\
Battery rating & $3.7 * 1650 \mathrm{mAH}$ \\
Value of $B P_{\text {threshold }}$ & $20 \%$ of battery rating \\
Battery drain range $\gamma$ & {$[0.1,10]$ mW at every one second } \\
$B_{t x} r x+B_{G P S} P$ Packet size & $1 \mathrm{~mW}$ at every one second \\
Assumed $P_{p}$ & 1000 Bytes \\
Value of $\Delta$ & 0.5 seconds \\
Value of $\delta_{t x}$ & 5 meters \\
Value of $L Q_{t h r e s h o l d}$ & $0.3 \mathrm{dBm}$ \\
\hline
\end{tabular}

TABLE I

Simulation PARAMETERS

$$
\begin{gathered}
\text { Region } 2=\pi *\left(r^{2}-2 * r * I C T_{\text {min_tx }}\right), \\
I C T_{\text {min_tx }}<R e g i o n 2 \leq\left(r-I C T_{\text {min_tx }}\right) \\
\text { Region } 3=\pi *\left(\left(2 * r * I C T_{\text {min_tx }}\right)-I C T_{\text {min_tx }}^{2}\right), \\
\left(r-I C T_{\text {min_tx }}\right)<\text { Region } 3 \leq r
\end{gathered}
$$

Data transmission is successful if data transmitting and receiving nodes are in the range for given $I C T_{m i n} t x$ duration. Proposed data forwarding protocol pseudocode to obtain TRRT is given in Algorithm 1. Required input parameters are explained in system model (section II). If the mobile nodes are chosen as relay nodes which are in the range of Region 1 , it may lead to negative progress [11], [19]. If relay nodes are chosen from Region3, there may be possibilities for node to move out of communication range, leading to link disconnection errors. Increase in link disconnections will increase number of retransmissions and TRRT. Therefore, first preference is for choosing relay node belonging to Region2, which neither leads negative progress or link disconnection errors. When mobile nodes are moving out of communication range, data sending node transmission power is increased by $\delta_{t x}$ to reduce number of link disconnections.

\section{Simulation Results and Discussion}

In this section we discuss the performance evaluation of proposed work. Each result point in the plots are the average 


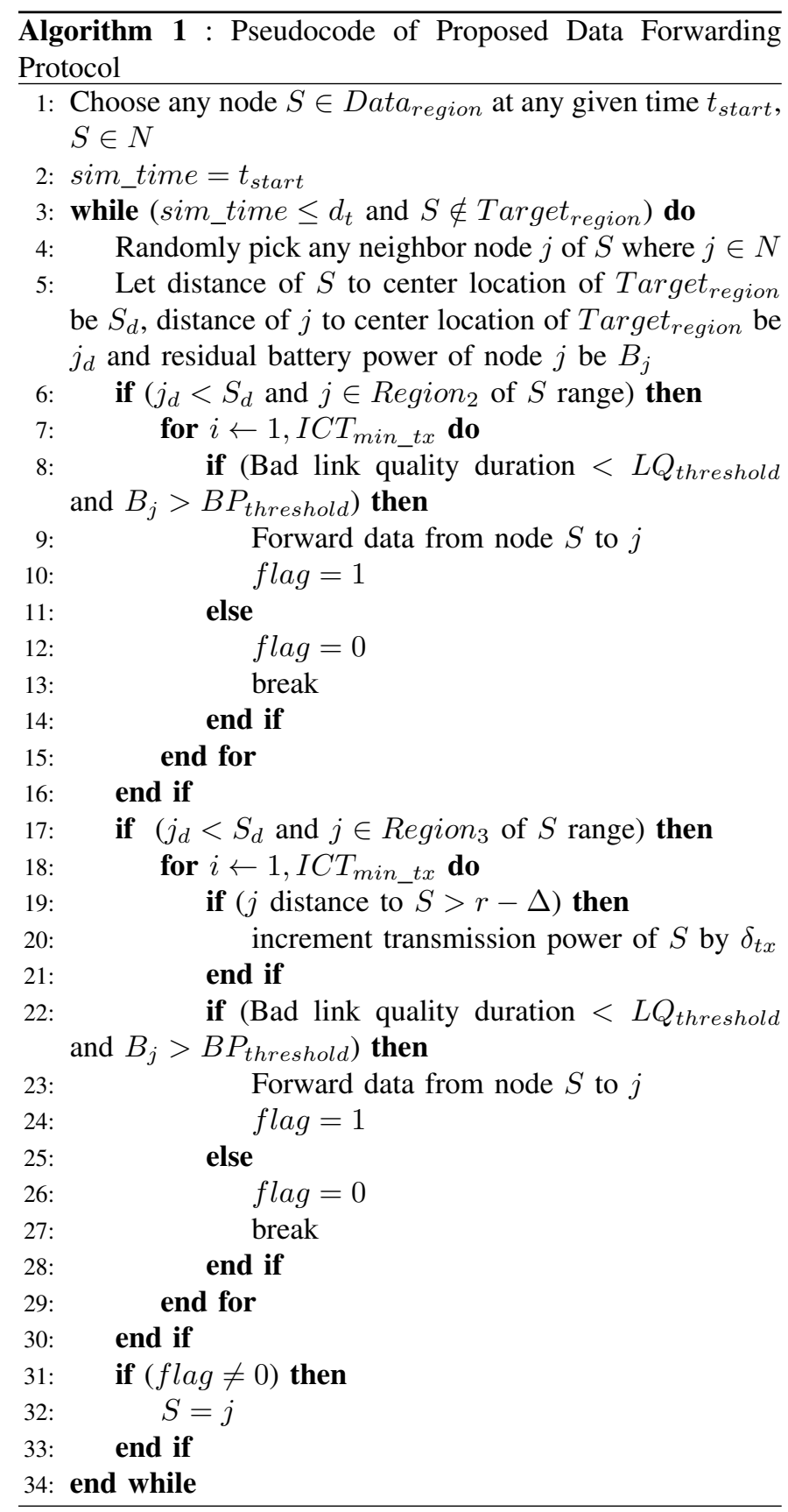

of thirty simulation runs and parameters considered for simulation are given in Table 1. Figure 2 shows $E_{N}$ with respect to varying $\alpha$ and $N$. From Figure 2, it is seen that, as $N$ increases, time gap to contact new neighbors in the network decreases. Considering low value for $\alpha$ causes highly dynamic mobile networks [3], [4] as the average velocity of mobile nodes increases and vice-versa. Therefore for $\alpha=0.2, E_{N}$ is low and for $\alpha=1.8, E_{N}$ is high (Figure 2). Figure 3 plots TRRT versus $I C T_{\text {min_tx }}$ for DTT-HWV and RP data forwarding protocols. We fixed $N$ to 500 and $I C T_{\text {min } \_t x}$ between 5 to 65 seconds (which is less than half of obtained range of mobile node). All mobile nodes are initialized with full battery power at the start of the simulation. It is shown in

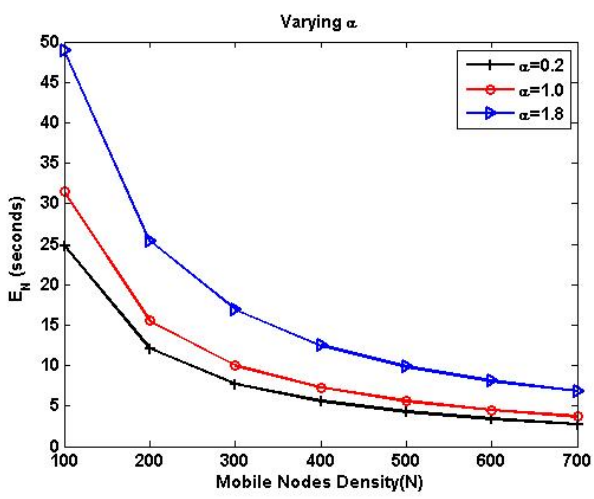

Fig. 2. $E_{N}$ values for varying $\alpha$ values

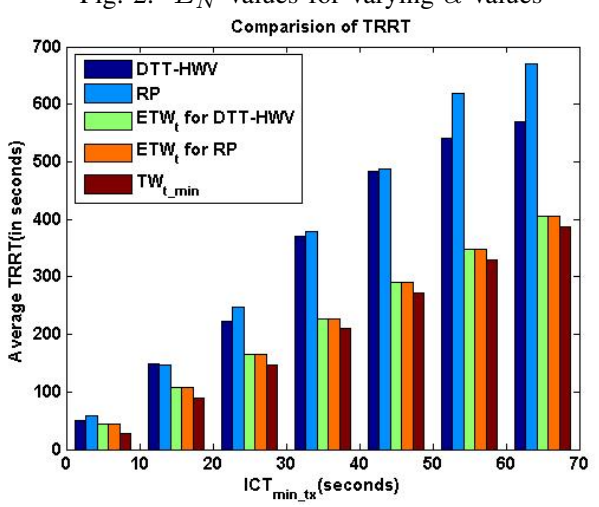

Fig. 3. Comparison of TRRT with varying $I C T_{m i n \_t x}$

Figure 3 that average TRRT values for DTT-HWV and RP forwarding methods are much higher than $E T W_{t}$ (equation (13)) and $T W_{t \_ \text {min }}$ (equation (14)). This is due to the effect of choosing a neighbor node randomly and unpredictable nature of human mobility patterns. It is also observed in Figure 3 that proposed protocol DTT-HWV gives better performance than $\mathrm{RP}$ data forwarding method for high $I C T_{\min t x}$ values. In RP data forwarding method there are chances for occurrence of backward progress (due to high $I C T_{\min } t x$ values) and also chances for choosing end nodes as data forwarding nodes, which increases network disconnection rate and TRRT. In DTT-HWV, backward progress and link disconnection rate are reduced by giving first preference to choose data forwarding mobile nodes that belongs to Region2. Figure 4 shows total battery power consumed during data forwarding $\left(B_{F d a t a}\right)$ (equation (7)) versus $I C T_{\min t x}$. From Figure 4, it is shown that, increase of transmission power $\left(\delta_{t x}\right)$ for the proposed protocol does not consume battery power more than RP data forwarding method. Figure 5 shows average number of hops used during data forwarding. Minimum number of hops $h$ (section III(C)) is represented by Min_no_hops. From Figure 5 performance of RP and DTT-HWV data forwarding methods in terms of average number of hops used, is not distinguishable. It is due to dynamic and unpredictable mobility nature of mobile phone users. Figure 6(a) and 6(b) shows low battery power and high path loss effect on TRRT. We 


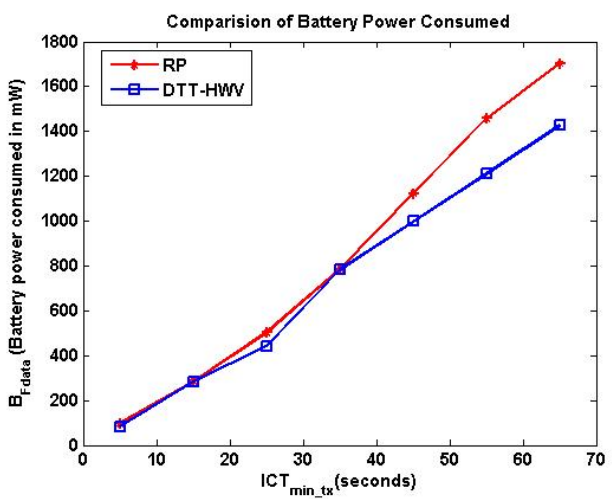

Fig. 4. Battery power consumed

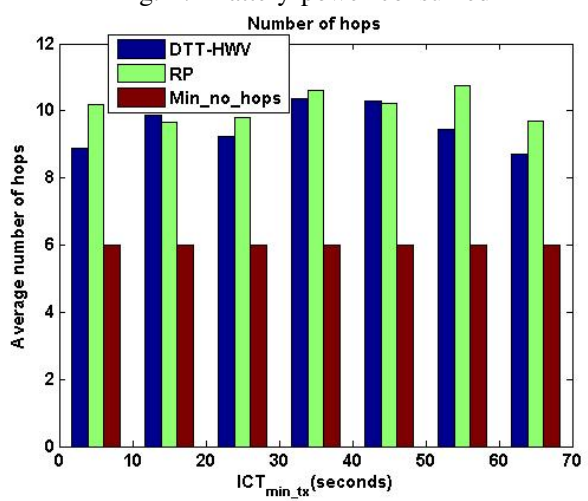

Fig. 5. Average number of hops
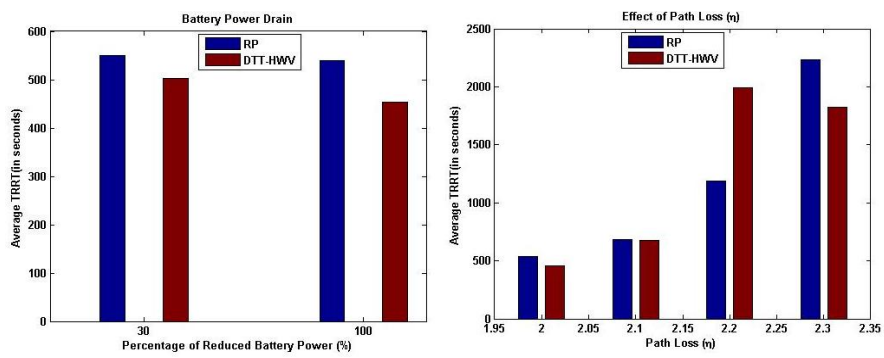

Fig. 6. (a) Battery power drain (b) Path loss effect on average TRRT

consider $N=400$ and $I C T_{\min } t x$ is 51 seconds. Figure 6(a) shows the average TRRT values when all nodes are initialised with full battery power $(100 \%)$ and randomly initialised with less then $30 \%$ of battery power at the start of simulation run. Very low residual battery power of mobile nodes increases data transmission failure rate, which leads to increase of average $T R R T$ for both proposed and RP data forwarding protocols (Figure 6(a)). Figure 6(b) shows that average TRRT increases with the increment of path loss exponent parameter $\eta$ for the proposed and RP data forwarding protocols.

\section{CONCLUSION}

For the given application, network and mobility model parameters, end-to-end waiting time (TRRT) to receive data is analysed for the proposed data forwarding protocol DTT-
HWV. DTT-HWV method has better performance as it reduces the number of link disconnections in the network and maintaining positive progress data forwarding towards target region. As Levy walk mobility model parameter $\alpha$ increases, expected time to get a neighbor node, $E_{N}$ also increases. Due to unpredictable and dynamic mobility nature of human walk pattern, end-to-end waiting time to forward data towards the target region is more than expected target reach time $E T W_{t}$. To overcome the effect of battery power drain and high path loss problems in opportunistic networks, mobile phone users walking duration and their density in the application area should be high enough. Our future work involves developing dynamic data forwarding protocol for OMPSN.

\section{REFERENCES}

[1] S. S. Kanhere, "Participatory sensing: Crowdsourcing data from mobile smartphones in urban spaces," IEEE International Conference on Mobile Data Management, pp. 3-6, 2011.

[2] L. Pelusi, A. Passarella, and M. Conti, "Opportunistic networking:data forwarding in disconnected mobile ad hoc networks," IEEE Communications Magazine, pp. 134-141, November 2006.

[3] I. Rhee, M. Shin, S. Hong, K. Lee, S. J. Kim, and S. Chong, "On the levy-walk nature of human mobility," IEEE/ACM TRANSACTIONS ON NETWORKING, vol. 19, no. 3, pp. 630-643, June 2011.

[4] B. Birand, M. Zafer, G. Zussman, and K.-W. Lee, "Dynamic graph properties of mobile networks under levy walk mobility," IEEE International Conference on Mobile Ad-Hoc and Sensor Systems, pp. 292-301, 2011.

[5] T. Karagiannis, J.-Y. L. Boudec, and M. Vojnovic, "Power law and exponential decay of inter contact times between mobile devices," MobiCom, pp. 183-194, September 2007.

[6] D. Chander, B. Jagyasi, U. B. Desai, and S. N. Merchant, "Layered data aggregation in cell-phone based wireless sensor networks," International Conference on Telecommunication, 2008.

[7] C. Bettstetter, G. Resta, and P. Santi, "The node distribution of the random waypoint mobility model for wireless ad hoc networks," IEEE TRANSACTIONS ON MOBILE COMPUTING, pp. 257-269, JulySeptember 2003.

[8] T. Camp, J. Boleng, and V. Davies, "A survey of mobility models for ad hoc network research," Wireless Communication and Mobile Computing (WCMC): Special issue on Mobile Ad Hoc Networking: Research, Trends and Applications, 2002.

[9] Networking Research Lab. Human mobility models downloads. [Online]. Available: http://research.csc.ncsu.edu/netsrv/

[10] D. Karamshuk, C. Boldrini, M. Conti, and A. Passarella, "Human mobility models for opportunistic networks," IEEE Communications Magazine, pp. 157-165, December 2011.

[11] M. Mauve and J. Widmer, "A survey on position-based routing in mobile ad hoc networks," IEEE Network, pp. 30-39, November/December 2001 2001.

[12] I. Stojmenovic, "Position-based routing in ad hoc networks," IEEE Communications Magazine, pp. 128-134, July 2002.

[13] R. Nelson and L. Kleinrock, "The spatial capacity of a slotted aloha multihop packet radio network with capture," IEEE TRANSACTIONS ON COMMUNICATIONS, pp. 684-694, June 1984.

[14] W. C. Y. Lee, Wireless and Cellular Telecommunication. McGrawHill, 2006.

[15] G. M. Viswanathan, S. V. Buldyrev, S. Havlin, M. G. E. da Luzk, E. P. Raposok, and H. E. Stanley, "Optimizing the success of random searches," NATURE, vol. 401, pp. 911-914, October 1999.

[16] G. Perrucci, F. Fitzek, and J. Widmer, "Survey on energy consumption entities on the smartphone platform," Vehicular Technology Conference (VTC Spring), 2011 IEEE, 2011.

[17] J. F. Kurose and A. K. W. Ross, Computer Networking: A Top-Down Approach. Addison-Wesley, 2010.

[18] Wikipedia. Preferred walking speed. [Online]. Available: http://en. wikipedia.org/wiki/Preferred_walking_speed

[19] H. Takagi and L. Kleinrock, "Optimal transmission ranges for randomly distributed packet radio terminals," IEEE TRANSACTIONS ON COMMUNICATIONS, vol. 32, no. 3, pp. 246-257, March 1984. 